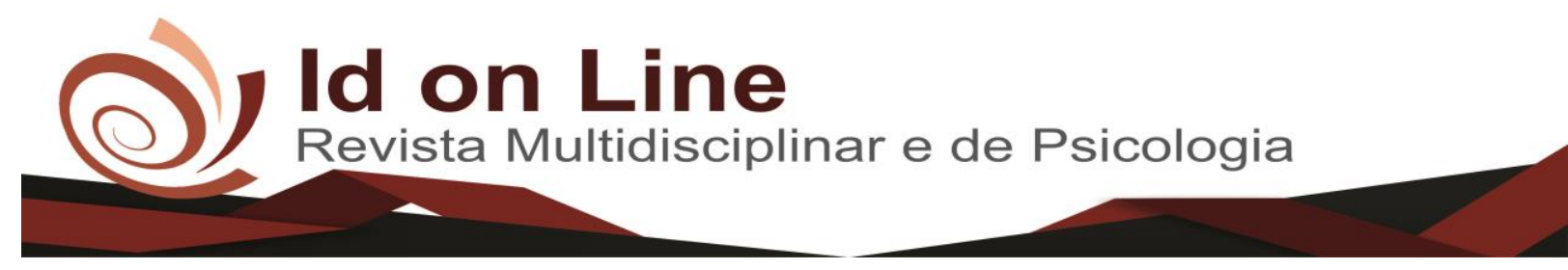

Artigo de Revisão

\title{
Uso do Laser de baixa intensidade no tratamento da Alopecia Androgenética: Uma Revisão Bibliográfica
}

\author{
Luciana Bárbara Pereira da Silva ${ }^{1}$; Belúzia Almeida Santos ${ }^{2}$
}

\begin{abstract}
Resumo: A alopecia androgenética se caracteriza pela perda parcial ou total dos fios e/ou pelos. Estes são transformados em velos (fios mais curtos, finos e com menos pigmento). A perda de cabelos provocada pela alopecia androgenética resulta na piora da qualidade de vida e bem-estar de quem a possui. Podem-se associar a isso quadros de depressão, ansiedade e estresse. Este estudo tem como objetivo analisar a eficácia do laser de baixa intensidade como forma de tratamento para alopecia androgenética através de uma revisão bibliográfica. O laser de baixa intensidade utilizado no tratamento da alopecia androgenética constitui-se na aplicação de feixes luminosos sobre o couro cabeludo para o aumento da textura e espessura dos fios. Analisando os estudos dos autores que citaram o uso do laser de baixa intensidade no tratamento da alopecia androgenética, percebe-se uma divergência de pensamentos. Foram encontradas poucas fontes de estudo que abordassem o uso do laser de baixa intensidade para o tratamento da alopecia androgenética, sendo este um tema que ainda possui muitos elementos a serem estudados a fim de se compreender de forma mais clara todas as etapas do desenvolvimento da alopecia e a influência do laser no seu tratamento.
\end{abstract}

Descritores: Alopecia androgenética, cabelo, laserterapia.

\section{Use of Low-Level Laser in the Androgenetic Alopecia Treatment: A Bibliographic Review}

\begin{abstract}
Androgenetic alopecia is characterized by partial or total loss of strands and/or hairs. These are transformed into veils (shorter, thinner and less pigmented strands). The loss of hair caused by androgenetic alopecia results in worsening of the life quality and well-being of those who own it. Pictures of depression, anxiety and stress can be associated with this. This study aims to analyze the efficacy of low intensity laser as a treatment form for androgenetic alopecia through a literature review. The low intensity laser used in the treatment of androgenetic alopecia is the application of light beams onto the scalp to increase the texture and thickness of the strands. Analyzing the studies of the authors who mentioned the use of the low intensity laser in the treatment of androgenetic alopecia, it is perceiveid a divergence of thoughts. Few sources of study have been found to address the use of low intensity laser for the treatment of androgenetic alopecia, which is a subject that still has many elements to be studied in order to understand more clearly all stages of the development of alopecia and the influence of the laser in its treatment.
\end{abstract}

Keywords: Androgenetic alopecia, hair, laser therapy.

\footnotetext{
${ }^{1}$ Graduanda do Curso Superior de Tecnologia em Estética e Cosmética da Faculdade Independente do Nordeste. Contato: luciana-90@ hotmail.com;

${ }^{2}$ Enfermeira especialista em Metodologia para o Ensino Superior e EAD e, em Atenção Dermatológica em Enfase em Estética e Cosmetologia. Docente nas graduações de Enfermagem e Tecnólogo em Estética e Cosmética da Faculdade Independente do Nordeste - FAINOR.
} 


\section{Introdução}

A preocupação com os cabelos é algo compartilhado entre homens e mulheres. O ser humano atribui aos cabelos grande importância, pois eles configuram uma imagem pessoal a ser transmitida, mostrando a personalidade da pessoa. O cabelo é um símbolo de beleza muito valorizado em todos os tempos e culturas (SANTOS; ALMEIDA; MOSER, s.d.).

A mídia veicula cabelos fartos e bem cuidados como o ideal de beleza almejado pelo indivíduo. Sendo assim, a perda excessiva dos cabelos configura em uma relação negativa na autoestima de ambos os sexos, prejudicando suas relações interpessoais e sociais (SILVA, 2011).

No ano de 460 a.C., Hipócrates, o pai da medicina, associou a queda de cabelos em humanos com a perda de pelos ocorrido nas raposas. A palavra grega 'alopekia' significa "sarna em raposa" e foi utilizada por Hipócrates para se referir a essa enfermidade. Ele também observou que eunucos e crianças não apresentavam este problema. Mais recentemente, na década de 1940, os estudos científicos realizados por J. B. Hamilton demonstraram que o hormônio testosterona só começava a ser produzido pelo organismo humano após a puberdade e que a presença deste hormônio, em maior ou menor grau, estava diretamente relacionada à queda de cabelos. Esta descoberta comprovou a observação de Hipócrates a respeito da não manifestação deste problema em crianças e eunucos, pois, como é sabido, a testosterona é produzida nos testículos. Em outras palavras, como os eunucos não possuem testículos e as crianças ainda não alcançaram a idade em que a testosterona é produzida, estes não apresentam alopecia (WEIDE; MILÃO, s.d.).

Devido a uma alteração no ciclo do cabelo, ocorre uma miniaturização dos fios, tornando-se finos e mais curtos. Este problema afeta o folículo piloso e ocorre pela conjunção de fatores genéticos e ambientais. Seu principal efeito negativo é o psicológico, podendo causar até mesmo depressão (REBELO, 2015). A alopecia que mais frequentemente acomete os homens e mulheres é a androgenética (calvície) e por este motivo foi escolhida como objeto de estudo desta pesquisa. A calvície masculina é caracterizada pela perda de cabelos na coroa do couro cabeludo e na região frontal (entradas). A queda de cabelos é geneticamente determinada e andrógeno dependente, ou seja, possui características próprias do sexo masculino. Apesar de não provocar danos à saúde, a queda dos cabelos pode prejudicar a qualidade de vida da pessoa afetada (SILVA; PATRICIO; PAULA, s.d.). Na alopecia feminina a queda de cabelo 
geralmente ocorre após a terceira década de vida piorando progressivamente após a menopausa e caracterizando-se por afinamento difuso dos fios. Em seu estágio inicial pode ocorrer queda de cabelo seguido de uma diminuição da densidade do fio. A grande maioria das mulheres relata negativamente a interferência da queda em sua autoestima e problemas sociais (MULINARIBRENNER; SEIDEL; HEPP, 2011).

Este estudo traz como objetivos: analisar a eficácia do laser de baixa intensidade como forma de tratamento para a alopecia androgenética através de uma revisão bibliográfica, utilizando artigos que abordam os efeitos do laser de baixa intensidade neste tratamento e também servir como referência de estudos sobre o assunto. O cabelo é considerado um atributo de beleza e é supervalorizado pelos indivíduos; sua perda pode ocasionar consequências psicológicas negativas como a depressão, baixa autoestima, ansiedade e stress. A busca por informações a respeito de tratamentos alternativos para essa patologia incentivou a elaboração deste trabalho. Sendo a alopecia androgenética uma disfunção estética que prejudica a qualidade de vida de quem a possui, a importância desta análise se dá por reunir estudos científicos que abordam a eficácia do laser de baixa potência como meio de terapia para estimular o crescimento capilar e retardar a progressão da queda.

\section{Metodologia}

O presente estudo trata-se de uma revisão bibliográfica. A pesquisa bibliográfica representa um importante instrumento utilizado para a construção de um processo de investigação, sua finalidade é colocar o pesquisador em contato com o que já se produziu a respeito do seu tema (GIL, 2010; LAKATOS; MARCONI, 2011).

A revisão bibliográfica representa esclarecimentos científicos, por meio de conhecimentos conceituados, acessíveis a partir de uma temática e contribui para o pesquisador adquirir informações para sua pesquisa e propor seu ponto de vista de acordo com o que foi descrito pelo autor.

Foram utilizados como fontes de pesquisa artigos científicos encontrados em sites acadêmicos e revistas científicas. As palavras-chaves usadas para busca foram: alopecia androgenética, cabelo e laserterapia. Teve como critérios de inclusão artigos científicos em língua portuguesa divulgados com caráter científico de 2006 a 2017 e tendo como finalidade 
caracterizar a alopecia e abordar o uso do laser como tratamento alternativo. Os critérios de exclusão foram artigos sem caráter científico e/ou em língua estrangeira.

Foi criado um quadro contendo as seguintes informações: título, autor, ano, categoria, suporte, tipo de pesquisa e objetivos. Logo após a discussão dos resultados apresentados no quadro com embasamento científico.

\section{Alopecia}

A alopecia pode ser caracterizada pela falta ou diminuição de pelos e/ou cabelos. Há diversos tipos de alopecia, por exemplo: a androgenética, que é uma das mais conhecidas, e a areata, causada por predisposição genética e agravada por fatores como o estresse e doenças autoimunes (SUCAR; SOUGEY; NETO, 2002 apud WEIDE; MILÃO, s.d.). A alopecia areata é uma afecção crônica de origem desconhecida, podendo ocorrer por diferentes causas. Não destrói o folículo, apenas interrompe a síntese do cabelo e por esse motivo pode ser reversível (RIVITTI, 2005 apud CAVALCANTI, 2015). A alopecia vem sendo estudada a muitos anos e cada vez mais indústrias cosméticas investem em avançadas pesquisas no intuito de entender mais sobre o assunto e tentar retardar esse processo que afeta diretamente a qualidade de vida de homens e mulheres, sendo mais frequente no sexo masculino. Atualmente muitos indivíduos são acometidos pela calvície e se tem poucos recursos para seu tratamento (PERES, 2012 apud CAVALCANTI, 2015).

\section{Alopecia feminina}

Segundo Cotterill (1992 apud JÚNIOR, 2006) e Sabatovich (2004 apud JÚNIOR, 2006), a perda de cabelos nas mulheres ocorre mais comumente em um período próximo à menopausa, por volta dos 40 anos, mas pode surgir em outras fases da vida. É nessa fase em que ocorre a menopausa que os cabelos se tornam enfraquecidos, finos e caem ocasionando a rarefação capilar.

A testosterona na mulher é sintetizada pelas glândulas supra-renais e pelos ovários. Uma enzima chamada 5-alfa-redutase contida na matriz do bulbo capilar transforma a testosterona em dehidrotestosterona, que ao penetrar no folículo o enfraquece e com isso é acelerada a queda 
dos fios. Quando a quantidade de testosterona na corrente sanguínea é elevada (maior que 1\%), isso pode provocar alterações no pelo e na pele. Os níveis de estrógenos diminuem na menopausa e havendo predisposição genética pode ocasionar calvície (SABATOVICH, 2004 apud JÚNIOR, 2006).

\section{Alopecia masculina}

A alopecia androgenética masculina geralmente tem início após a adolescência, com a queda dos cabelos começando pela região temporal podendo chegar até ovértex, o ponto mais alto do crânio. O seu avanço pode variar, porém quanto mais cedo se inicia, geralmente é mais abundante. $14 \%$ dos meninos entre 15 e 17 anos podem apresentar a calvície precocemente (TRANCIK et al apud MULINARI-BRENNER; SEIDEL; HEPP, 2011).

No folículo piloso a ação dos andrógenos ocorre possivelmente na papila dérmica com a ligação de receptores específicos (OLSEN et al apud MULINARI-BRENNER; SEIDEL; HEPP, 20011).

O desenvolvimento da alopecia androgenética e a miniaturização dos folículos se dá pelo andrógeno di-hidrotestosterona, um metabólito da testosterona que também é responsável pela patogenia da neoplasia de próstata, hiperplasia prostática benigna, acne vulgar e hirsutismo (TRÜEB, 2002; OLSEN et al, 2005; KAUFMAN, 1996 apud MULINARI-BRENNER; SEIDEL; HEPP, 2011).

A excessiva produção de andrógenos no folículo pilossebáceo ou uma resposta exagerada dos receptores de andrógenos podem ser responsáveis pelo acometimento da calvície, embora, na maioria dos homens, esses andrógenos se apresentam em quantidades normais (CHEN; ZOUBOULIS, 2009 apud MULINARI-BRENNER; SEIDEL; HEPP, 2011).

\section{Alopecia androgenética}

A alopecia mais comum entre homens e mulheres é a androgenética, na qual ocorre uma alteração no ciclo do cabelo transformando-o em velo (fios mais curtos, finos e com menos pigmento) (TRÜEB, 2002; KRAUSE, 2006 apud MULINARI-BRENNER; SEIDEL; HEPP, 2011). 
O dia a dia corrido, de modo geral, acaba por afetar negativamente a saúde do indivíduo alterando seu estado emocional e provocando estresse. Esta é uma das principais causas da perda dos cabelos, além de outros fatores como o uso de medicamentos, disfunção hormonal ou traumas. Segundo a definição de Orentreich, indivíduos com a presença de androgênios possuem folículos predispostos à miniaturização, gerando a calvície (SILVA; PATRÍCIO; PAULA, s.d.).

Essa afecção ocorre em ambos os sexos. No homem essa miniaturização pode ocorrer após a puberdade em qualquer idade - sendo mais aparente por volta dos 17 anos - e nas mulheres endocrinologicamente normais por volta dos 25 a 30 anos de idade (ROGERS; AVRAM, 2008; ESPINOZA, 2008 apud WEID; MILÃO, s.d.). “É de origem genética (alopecia androgenética) e pode estar associada às doenças endócrinas, onde há predominância da testosterona" (JÚNIOR, 2006, p. 197).

\section{Estrutura do cabelo}

O cabelo tem seu desenvolvimento no folículo piloso e na sua base as células se multiplicam passando por processos que transformam seu citoplasma em um material fibroso, o cabelo. O folículo tem composição epitelial como a bainha interna e externa, matriz e haste e composição dérmica como a bainha dérmica e papila dérmica (CALLAND, 2007 apud SILVA; PATRICIO; PAULA, s.d.).

No couro cabeludo o número de folículos é cerca de cem mil. O folículo começa a se desenvolver primeiramente com uma invaginação da epiderme em direção à derme formando um canal profundo que fica acima do tecido subcutâneo (HALAL, 2011 apud SILVA; PATRICIO; PAULA, s.d.).

Na região mais profunda do folículo encontra-se o bulbo e nele a matriz germinativa, que recobre a papila dérmica onde situam-se os fibroblastos que determinam o tamanho do pelo, pois controlam o número de células na matriz. Nessa matriz ocorre a multiplicação de células movendo-se em direção à superfície para formar o pelo. Esta região é altamente vascularizada (PEREIRA et al, 2001 apud SILVA; PATRICIO; PAULA, s.d.).

O cabelo é composto em sua maioria por queratina e possui três camadas principais (cutícula, córtex e medula). A cutícula é a camada mais externa do cabelo formada por células sobrepostas transparentes assemelhando-se a escamas. O córtex possui células queratinizadas 
onde se encontra a cor dos cabelos; ele é flexível e elástico. A medula, parte mais interna, é formada por fileiras de células lado a lado (HALAL, 2011 apud SILVA; PATRICIO; PAULA, s.d.).

\section{Ciclo de Crescimento do Cabelo}

O folículo possui um ciclo de desenvolvimento dividido em três fases: anágena, catágena e telógena. A fase anágena, em que o cabelo se desenvolve e cresce, pode ter duração de dois a seis anos, e se encontra em atividade mitótica da matriz. Já a fase catágena é a menor fase do ciclo e tem duração de duas a três semanas. Nela ocorre um declínio das divisões das células capilares e involução do folículo. $\mathrm{Na}$ fase telógena, o fio apresenta-se mais delgado e claro e tem duração de aproximadamente três meses. Há um estímulo da divisão celular das células-tronco para assim reiniciar o ciclo e o cabelo cai ou é empurrado para fora (MANSUR; GAMONAL, 2004 apud WIELEWSKI; SERRÃO; MOSER, s.d.).

\section{Impacto psicológico da alopecia androgenética}

A alopecia prejudica a vida de ambos os sexos, estando associada à baixa autoestima e a um índice maior de ansiedade, depressão e stress (GRIMALT, 2005; KONTOS, 2008 apud KLEINHANS, 2012). Alguns autores relacionaram avaliação pessoal, stress e queda de cabelos a um círculo vicioso. Estes explicam que a percepção que os indivíduos com alopecia têm de si mesmos e o modo como o problema é visto pela sociedade podem gerar depressão, ansiedade e stress, o que colabora com o aumento da queda enquanto o adequado cuidado quanto ao stress e o tratamento farmacológico e psicoterapêutico podem diminuí-la (ARCK et al 2004 apud KLEINHANS, 2012).

Segundo COSTA (2016), a alopecia androgenética, mesmo não prejudicando a saúde, pode agravar a autoestima dos homens e com isso provocar distúrbios psicológicos. Outro autor que apresenta a mesma opinião diz que a alopecia possui poucos efeitos físicos prejudiciais, porém, pode levar a consequências psicológicas negativas e até gerar depressão (REBELO, 2015). 
O cabelo apresenta grande importância tanto pela sua aparência jovial como pelo fato de ser considerado um fator de saúde e reconhecimento dos indivíduos. Sua perda, embora apresente poucos efeitos danosos sobre o organismo, pode gerar consequências psicológicas negativas levando a pessoa acometida a ter ansiedade e depressão. Foram realizados estudos relatando que o cotidiano das pessoas foi afetado pela influência dos problemas de autoconfiança e que as mesmas evitam sair de casa, prejudicando até no seu ambiente de trabalho (SILVA, 2011).

\section{Laser de baixa intensidade}

O laser é um aparelho que consiste de substâncias de origem líquida, sólida ou gasosa que fabricam um feixe de luz quando estimuladas por uma fonte de energia, usualmente chamado também por "raio laser". Estes podem ser classificados em: lasers de alta intensidade ou cirúrgicos, que possuem efeitos térmicos atuando no corte, na vaporização e homeostasia, e lasers de baixa intensidade ou terapêuticos, sendo utilizados para efeitos de bioestimulação, anti-inflamatório e analgesia (SILVA et al, 2007; BARROS et al, 2008 apud LINS et al, 2010).

O laser de baixa intensidade, por ter uma densidade baixa de energia, expõe as células ou tecidos a níveis baixos de luz vermelha se comparado a outras formas de laserterapia que são usadas para ablação, corte e coagulação térmica do tecido. A terapia com laser de baixa intensidade pode ser conhecida também por "laser frio", pois sua densidade de potência é menor do que a necessária para gerar aquecimento de tecido (AVCI et al, 2014; MUNCK; GAVAZZONI; TRUEB, 2014; TIN; WIWANITKIT, 2014 apud SILVA, M; SILVA, C., 2017).

A laserterapia de baixa intensidade (LTBI) ou baixa potência (reativo) é um termo genérico que define a aplicação terapêutica de lasers e diodos superluminosos monocromáticos, com potência relativamente baixa, para o tratamento de feridas abertas, lesões de tecidos moles, processos inflamatórios e dores associadas a várias etiologias, com dosagens consideradas baixas demais para efetuar qualquer aquecimento detectável nos tecidos irradiados (KITCHEN et al, 1998 apud MEZZALIRA; FREDERICO, 2007, p. 10).

Por ser uma doença muito comum, vem-se buscando vários tipos de tratamento para a alopecia, embora não se tenha encontrado resultados que sejam realmente satisfatórios. A terapia a laser vem sendo utilizada como tratamento alternativo para a prevenção da queda 
capilar e, além de prevenir, estimula o crescimento de cabelo na alopecia androgenética masculina e feminina (RANGWALA; RASHID, 2012 apud ANTONIO, JR.; ANTONIO, CR.; TRÍDICO LA, 2017).

Não se tem uma avaliação exata da ação desse mecanismo nos cabelos. É provável que ocorra a ativação da reentrada dos fios telógenos que estão em repouso, para a fase de proliferação (anágena), elevando a duração do ciclo e o crescimento durante essa fase e impedindo sua entrada no estágio de involução (catágena) (ANTONIO, J.; ANTONIO, C.; TRÍDICO L., 2017).

Os efeitos do laser fracionado $1550 \mathrm{~nm}$ foram avaliados por Kim et al na alopecia androgenética masculina e foi verificado um aumento da densidade e do crescimento do cabelo. Há a estimulação e regeneração de colágeno através da fotodermólise fracionada pelo comprimento de onda. A análise histológica comprovou a conversão dos folículos em fase de repouso para a de crescimento (KIM et al apud ANTONIO, JR.; ANTONIO, CR.; TRÍDICO LA, 2017).

O fato de o laser de baixa intensidade poder contribuir para o crescimento dos fios pode estar associado à capacidade da baixa potência da luz de estimular o bulbo capilar e não provocar termólise. O calor pode ser suficiente para estimular o crescimento do cabelo, pela diferenciação das células-tronco foliculares, indução indireta da angiogênese folicular e ao ciclo celular. Para o tratamento da alopecia androgenética o comprimento de onda indicado é o da faixa de $630 \mathrm{~nm}$ a $670 \mathrm{~nm}$ (AVCI et al, 2014; MUNCK; GAVAZZONI; TRUEB, 2014; TIN; WIWANITKIT, 2014 apud SILVA, M; SILVA, C., 2017).

Durante a estimulação do laser de baixa intensidade ocorre uma série de fatores que irão resultar na reversão de folículos dormentes para folículos em crescimento. Assim, temos a modulação de espécies reativas de oxigênio (ROS) e o aumento da produção de adenosina trifosfato (ATP); nas células tratadas, a indução de fatores de transcrição; aumento da velocidade de transporte de elétrons na cadeia respiratória pela mitocôndria, aumento da proliferação celular, oxigenação dos tecidose e fatores de crescimento (MUNCK; GAVAZZONI; TRUEB, 2014, AVCI et al, 2014 apud SILVA, M; SILVA, C., 2017).

O tratamento da alopecia utilizando-se o laser tem resultados satisfatórios relatados pelos pacientes, que devem iniciar a terapia até a fase intermediária do aparecimento da alopecia para que o tratamento seja mais eficaz. Os estudos demonstram que este tipo de tratamento é seguro para ambos os sexos, sendo relatadas melhoras no crescimento geral do cabelo, diminuição de sua perda, pelos mais espessos, couro cabeludo mais saudável e cabelos mais 
brilhosos após a laserterapia (AVCI et al, 2014; MUNCK; GAVAZZONI; TRUEB, 2014; LANZAFAME et al, 2014 apud SILVA, M; SILVA, C, 2017).

O laser de baixa intensidade utilizado no tratamento da alopecia androgenética constituise na aplicação de feixes luminosos sobre o couro cabeludo para o aumento da textura e espessura dos fios. Dentre seus efeitos estão o de possuir uma ação bioestimulante no processo de reparo tecidual, induzir atividade mitótica das células epiteliais e também dos fibroblastos, que serão estimulados a produzir colágeno, inibir a secreção de alguns mediadores químicos, modificar a densidade capilar, e incentivar a microcirculação local (LINS et al, 2010; MULINARI-BRENNER; SOARES, 2009 apud COSTA, 2016).

A terapia com laser é indicada tanto para disfunções estéticas como para tratamentos de vários processos patológicos, visto que a luz do laser sobre os tecidos aumenta o grau de atividade mitótica e biológica nas células, e a luz sendo um tipo de energia é essencial para manter a homeostase do organismo (MARTINS et al, 2013 apud CATELAN et al, 2016). "O incremento energético nas células com a ação do laser, induzem as células do folículo pilo sebáceo restabelecer suas funções, superando fatores inibidores do crescimento, devolvendo sua capacidade de produzir fios" (MARTINS et al, 2013 apud CATELAN et al, 2016, p. 480).

O laser age nas células periféricas da papila dérmica ativando suas células germinativas que têm a capacidade de regenerar e revitalizar o folículo piloso, atuando na elevação da vascularização, estímulo do crescimento do pelo e células-tronco no bulbo do folículo piloso, aporte de nutrientes e extensão da fase anágena (BORGES; SCORZA, 2016 apud LIMA et al, 2016). 
Quadro 1 - Material utilizado neste estudo

\begin{tabular}{|c|c|c|c|c|c|c|c|}
\hline $\mathbf{N}^{\circ}$ & TíTULO & AUTOR(ES) & $\begin{array}{c}\text { ANO DE } \\
\text { PUBLICAÇÃO }\end{array}$ & CATEGORIA & SUPORTE & TIPO DE PESQUISA & OBJETIVOS \\
\hline 1 & $\begin{array}{l}\text { A Utilizaçãoda Finasterida no } \\
\text { Tratamento da Alopecia } \\
\text { Androgenética }\end{array}$ & $\begin{array}{l}\text { WEIDE, A. } \\
\text { MILÃO, D. }\end{array}$ & s.d. & Artigo & Artigo & Revisão de literatura & $\begin{array}{l}\text { Realizar uma revisão de literatura sobre a utilização da } \\
\text { finasterida no tratamento da alopécia }\end{array}$ \\
\hline 2 & $\begin{array}{l}\text { Abordagens da Farmácia } \\
\text { Estética no Tratamento da } \\
\text { Alopecia Androgenética }\end{array}$ & $\begin{array}{l}\text { SILVA, M } \\
\text { SILVA, C }\end{array}$ & 2017 & Artigo & Revista & Revisão de literatura & $\begin{array}{l}\text { Caracterizar a alopecia androgenética como disfunção estética, } \\
\text { e realizar um levantamento dos tratamentos usando os } \\
\text { seguintes recursos estéticos: laser de baixa intensidade, } \\
\text { microagulhamento, carboxiterapia e } \\
\text { mesoterapia/intradermoterapia }\end{array}$ \\
\hline 3 & $\begin{array}{l}\text { Alopecia Feminina: Uma } \\
\text { abordagem do processo e } \\
\text { tratamentos não } \\
\text { convencionais aplicados a } \\
\text { esta patologia }\end{array}$ & $\begin{array}{l}\text { SANTOS, A } \\
\text { ALMEIDA, T }\end{array}$ & s.d. & Artigo & Artigo & $\begin{array}{l}\text { Qualitativa do tipo } \\
\text { exploratório } \\
\text { bibliográfica }\end{array}$ & $\begin{array}{l}\text { Abordar os processos e tratamentos não convencionais } \\
\text { aplicados à alopecia feminina }\end{array}$ \\
\hline 4 & $\begin{array}{l}\text { Análise Comparativa de } \\
\text { Técnicas de Massagem } \\
\text { Utilizadas em um Protocolo } \\
\text { de Tratamento para Queda } \\
\text { Capilar }\end{array}$ & $\begin{array}{l}\text { WIELEWSKI, C } \\
\text { SERRÃO, C }\end{array}$ & s.d. & Artigo & Artigo & $\begin{array}{l}\text { Qualitativa do tipo } \\
\text { exploratória descritiva }\end{array}$ & $\begin{array}{l}\text { Fazer uma análise comparativa da eficácia do uso da massagem } \\
\text { e os princípios ativos no couro cabeludo em um protocolo de } \\
\text { tatamento para queda capilar }\end{array}$ \\
\hline 5 & $\begin{array}{l}\text { Calvície Feminina: } \\
\text { Classificação Proposta }\end{array}$ & JÚNIOR, F & 2006 & Artigo & $\begin{array}{l}\text { Revista } \\
\text { Brasileira de } \\
\text { Cirurgia } \\
\text { Plástica }\end{array}$ & Caso Clínico & $\begin{array}{l}\text { Apresentar uma classificação em seis graus, mais simples, } \\
\text { abrangente e didática, facilitando o diagnóstico clínico e } \\
\text { priorizando, dessa forma, uma denominação pré-operatória } \\
\text { mais objetiva para todos os tipos de calvície feminina } \\
\text { encontrados na clínica diária. }\end{array}$ \\
\hline
\end{tabular}




\begin{tabular}{|c|c|c|c|c|c|c|c|}
\hline 6 & $\begin{array}{l}\text { Efeitos bioestimulantes do } \\
\text { laser de baixa potência no } \\
\text { precesso de reparo }\end{array}$ & LINS, R et al. & 2010 & Artigo & $\begin{array}{l}\text { Revista Anais } \\
\text { Brasileiros de } \\
\text { Dermatologia }\end{array}$ & Relato de Caso & $\begin{array}{l}\text { Estudar o fenômeno da bioestimulação e destacar os principais } \\
\text { efeitos bioestimulantes do laser de baixa potência na reparação } \\
\text { tecidual }\end{array}$ \\
\hline 7 & $\begin{array}{l}\text { Entendendo a Alopecia } \\
\text { Androgenética }\end{array}$ & $\begin{array}{l}\text { MULINARI- } \\
\text { BRENNER, F } \\
\text { SEIDEL, G } \\
\text { HEPP, T }\end{array}$ & 2011 & Artigo & $\begin{array}{l}\text { Revista Surgical } \\
\& \text { Cosmetic } \\
\text { Dermatology }\end{array}$ & Revisão de literatura & $\begin{array}{l}\text { Aumentar a cobertura do couro cabeludo e retardar a } \\
\text { progressão da queda. Este artigo aborda de forma didática a } \\
\text { fisiopatogenia da alopecia androgenética, sua correlação com } \\
\text { as manifestações clínicas e resposta terapêutica }\end{array}$ \\
\hline 8 & $\begin{array}{l}\text { Laserterapia de Baixa } \\
\text { Intensidade Revisão de } \\
\text { Literatura }\end{array}$ & $\begin{array}{l}\text { MEZZALIRA, J } \\
\text { FREDERICO, I }\end{array}$ & 2007 & Artigo & Artigo & Revisão de literatura & Discutir a eficácia do laser através de um levantamento literário \\
\hline 9 & $\begin{array}{l}\text { Medicamentos } \\
\text { Antiandrógenos de Uso Oral } \\
\text { para o Tratamento da } \\
\text { Alopecia Androgênica }\end{array}$ & SILVA, R & 2011 & Artigo & Monografia & Revisão de literatura & $\begin{array}{l}\text { Realizar um estudo de revisão da literatura científica sobre as } \\
\text { terapêuticas aplicadas no tratamento antiandrógeno, por via } \\
\text { oral, da alopecia androgênica masculina e feminina. }\end{array}$ \\
\hline 10 & $\begin{array}{l}\text { Microagulhamento para } \\
\text { tratamento da alopecia } \\
\text { androgenética masculina }\end{array}$ & COSTA, A & 2016 & Artigo & Monografia & Revisão de literatura & $\begin{array}{l}\text { Descrever a utilização do microagulhamento no tratamento da } \\
\text { alopecia androgenética masculina, compreender a estrutura e } \\
\text { ciclo capilar, abordar distúrbios inerentes ao folículo piloso, } \\
\text { conhecer a alopecia androgenética e os tratamentos indicados. }\end{array}$ \\
\hline 11 & $\begin{array}{l}\text { Novas Estratégias para o } \\
\text { Tratamento da Alopecia }\end{array}$ & REBELO, A & 2015 & Artigo & $\begin{array}{l}\text { Dissertação de } \\
\text { mestrado }\end{array}$ & Revisão de literatura & Não especificado \\
\hline
\end{tabular}




\begin{tabular}{|c|c|c|c|c|c|c|c|}
\hline 12 & $\begin{array}{l}\text { O uso do laser de baixa } \\
\text { potência no estímulo do } \\
\text { crescimento capilar em } \\
\text { homens com alopecia } \\
\text { androgenética entre } 25 \text { e } 35 \\
\text { anos }\end{array}$ & $\begin{array}{l}\text { CATELAN, A } \\
\text { KOBAYASHI, G } \\
\text { PEREIRA, L }\end{array}$ & 2016 & Artigo & $\begin{array}{l}\text { Revista } \\
\text { científica do } \\
\text { Unisalesiano }\end{array}$ & Caso clínico & $\begin{array}{l}\text { Estimular o crescimento capilar, utilizando como método } \\
\text { terapêutico o laser vermelho de baixa potência, com } \\
\text { comprimento de onda de } 660 \mathrm{~nm}\end{array}$ \\
\hline 13 & $\begin{array}{l}\text { Stress e Raiva em Mulheres } \\
\text { com Alopecia Androgenética }\end{array}$ & KLEINHANS, A & 2012 & Artigo & Dissertação & Revisão bibliográfica & $\begin{array}{l}\text { Avaliar o nível de stress e a expressão da raiva, tanto para } \\
\text { dentro como para fora, em uma amostra de mulheres com } \\
\text { alopecia androgenética (AA). }\end{array}$ \\
\hline 14 & $\begin{array}{l}\text { Terapia Capilar para o } \\
\text { Tratamento da alopecia } \\
\text { androgenética masculina e } \\
\text { alopecia areata }\end{array}$ & $\begin{array}{l}\text { SILVA, E } \\
\text { PATRICIO, M }\end{array}$ & s.d. & Artigo & Artigo & Caso clínico & $\begin{array}{l}\text { Descrever os resultados do tratamento com uso da Terapia } \\
\text { Capilar, protocolo específico elaborado pela empresa Extratos } \\
\text { da Terra }\end{array}$ \\
\hline 15 & $\begin{array}{l}\text { Tratamento da Alopecia } \\
\text { Androgenética- Associação } \\
\text { de Laser Erbium Glass } \\
\text { 1550nm e Infiltração de } \\
\text { Ativos }\end{array}$ & $\begin{array}{l}\text { ANTONIO, J } \\
\text { ANTONIO, C } \\
\text { TRÍDICO, L }\end{array}$ & 2017 & Artigo & $\begin{array}{l}\text { Revista Surgical } \\
\& \text { Cosmetic } \\
\text { Dermatology }\end{array}$ & Caso clínico & $\begin{array}{l}\text { Avaliar o resultado do uso do laser de Erbium Glass 1550nm } \\
\text { associado à infiltração de ativos (minoxidil, finasterida, fatores } \\
\text { de crescimento e complexo vitamínico) para tratamento da } \\
\text { alopecia androgenética masculina e feminina. }\end{array}$ \\
\hline 16 & $\begin{array}{l}\text { Tratamento de Alopécia } \\
\text { Androgenética com o uso de } \\
\text { Laser de Baixa Potência e } \\
\text { Dermotonia (Vacuoterapia) } \\
\text { Associado a Argiloterapia e } \\
\text { Óleos Essenciais }\end{array}$ & LIMA, J et al. & 2016 & Artigo & $\begin{array}{l}\text { Revista Anais } \\
2016\end{array}$ & $\begin{array}{l}\text { Investigativo e } \\
\text { exploratório, através } \\
\text { de um estudo de caso }\end{array}$ & $\begin{array}{l}\text { Demonstrar a eficácia da Terapia Capilar no tratamento da } \\
\text { Alopécia Androgenética utilizando a Argiloterapia e Óleos } \\
\text { Essenciais comparando os resultados do Laser Red de Baixa } \\
\text { Potência com Dermotonia. }\end{array}$ \\
\hline 17 & $\begin{array}{l}\text { Protocolos de tratamento da } \\
\text { alopecia: uma revisão }\end{array}$ & CAVALCANTI, C & 2015 & Artigo & Artigo & $\begin{array}{l}\text { Pesquisa exploratória } \\
\text { bibliográfica }\end{array}$ & $\begin{array}{l}\text { Realizar um estudo de revisão sistêmica da literatura científica, } \\
\text { sobre o tratamento da alopecia. }\end{array}$ \\
\hline
\end{tabular}

Fonte: Dados da pesquisa. 


\section{Discussão}

A partir dos 17 artigos utilizados no quadro desta pesquisa, verificou-se que oito deles falam sobre o laser e seis abordam o uso do laser de baixa intensidade no tratamento da alopecia androgenética, sendo que desses seis artigos, três utilizaram o laser em seus estudos e o restante apenas o citaram.

Dos artigos em que o laser foi utilizado, dois deles empregaram associações com outros tratamentos. Segundo Antonio, J; Antonio, C; Trídico, L (2017), foi avaliado o uso do laser de Erbium Glass $1550 \mathrm{~nm}$ associado à infiltração de ativos como o minoxidil, finasterida, fatores de crescimento e complexo vitamínico para a terapia da alopecia androgenética em ambos os sexos. A maioria dos pacientes ficou satisfeita com o tratamento, sendo que os mais satisfeitos realizaram mais sessões. Foi relatado que esta associação é segura e eficaz para o tratamento da alopecia androgenética.

De acordo com Lima, $\mathrm{J}$ et al o laser utilizado foi associado à argiloterapia e óleos essenciais, também foi utilizada dermotonia. Foi observada uma diferença nos resultados: nas duas voluntárias que se submeteram ao tratamento, uma foi tratada com o laser e a outra utilizou a dermotonia. Verificou-se ao final do tratamento que nas duas voluntárias o resultado tanto da dermotonia quanto do laser e associações foram eficazes no surgimento de novos fios, tendo diâmetros reduzidos e sem pigmentos.

O uso do laser de baixa intensidade, com comprimento de onda de $660 \mathrm{~nm}$ utilizado por CATELAN et al (2016) com objetivo de promover o crescimento capilar, entre outros, mostrouse seguro e sem nenhum efeito colateral, contribuindo para o aparecimento de novos fios e maior permanência do pelo no folículo, diminuindo sua queda e beneficiando a autoestima de seus voluntários.

Analisando os estudos dos três autores que citaram o uso do laser de baixa intensidade no tratamento da alopecia androgenética, percebe-se uma divergência de pensamentos. Segundo Costa (2016), não foram encontradas respostas satisfatórias nos estudos com aparelhos de laser para a alopecia androgenética, e, apesar de ser aprovado pela entidade reguladora americana FDA (Food and Drug Administration), foi testado apenas para a segurança do usuário e não para seu resultado.

Já de acordo com Rebelo (2015), o tratamento utilizando o laser de baixa intensidade para a alopecia androgenética é especialmente indicado para aqueles que não tiveram resultados 
positivos ou apresentaram efeitos colaterais com o uso de medicamentos e têm preferência por tratamentos não cirúrgicos.

A terapêutica com o laser de baixa intensidade pode ser uma possível fonte de estimulação dos fios, seu mecanismo de ação ainda não é totalmente conhecido, mas acreditase que atua nas mitocôndrias, podendo mudar o metabolismo celular, elevando a produção de adenosina trifosfato (ATP), a proliferação das células, fatores de crescimento e oxigenação dos tecidos. Supõe-se que esse laser faz com que os folículos em fase telógena reentrem em fase anágena prolongada (REBELO, 2015).

O uso do laser é descrito por vários autores que utilizam diversos comprimentos de onda e equipamentos de variados tipos. De um modo geral foram comprovados pelos estudos a segurança desse tratamento tanto para homens quanto para mulheres, que relataram melhoras no crescimento geral do cabelo, espessura, saúde do couro cabeludo e redução da perda dos fios (SILVA, M; SILVA, C., 2017).

\section{Considerações Finais}

A alopecia androgenética, apesar de não prejudicar a saúde dos indivíduos, pode levar a consequências psicológicas negativas. As buscas por seu tratamento são crescentes e terapias alternativas à terapêutica farmacológica e cirurgia vêm sendo estudadas visando uma melhora do bem-estar do paciente/cliente como um todo (corpo e mente).

Foram encontradas poucas fontes de estudo que abordassem o uso do laser de baixa intensidade para o tratamento da alopecia androgenética. Alguns autores dizem ser este um método eficaz e seguro, outros falam que é preciso mais conhecimento sobre a fisiopatogenia da alopecia androgenética para uma melhor indicação de tratamento.

Este é um tema que ainda possui muitos elementos a serem estudados, a fim de se compreender de forma mais clara todas as etapas do desenvolvimento da alopecia e a influência do laser no seu tratamento. Neste sentido, espera-se que este estudo seja capaz de gerar outros que se aprofundem mais sobre o assunto. 


\section{Referências}

ANTONIO, J.; ANTONIO, C.; TRÍDICO, L. Tratamento da alopecia androgenética: associação de laser Erbium Glass 1550nm e infiltração de ativos. São José do Rio Preto-SP, 2017, p. 19-23. Disponível em: <http://www.surgicalcosmetic.org.br/detalhe-artigo/544/Tratamento-da-alopecia-androgenetica-associacao-de-laser-Erbium-Glass-1550nm-e-infiltracao-de-ativos>. Acessado em: 24/05/2018.

CATELAN, A.et al. O uso do laser de baixa potência no estímulo do crescimento capilar em homens com alopecia androgenética entre 25 e 35 anos. Disponível em: $<\mathrm{http} / / / \mathrm{www}$. salesianolins.br/universitaria/artigos/no15/artigo88.pdf $>$. Acessado em: 24/05/2018.

CAVALCANTI, C. Protocolos de tratamento da alopécia: uma revisão. Disponível em: <http://dspace.bc.uepb.edu.br/jspui/bitstream/123456789/8683/1/PDF\%20-

$\% 20$ Carla\%20Pereira\%20Cavalcanti.pdf $>$. Acessado em: 24/05/2018.

COSTA, A., Microagulhamento para tratamento da alopecia androgenética masculina. Disponível em: <http://www.ccecursos.com.br/img/resumos/microagulhamento-para-tratamento-da-alopeciaandrogen-tica-masculina-tcc---aline-fransuely-ribeiro-da-costa.pdf $>$. Acessado em: 24/05/2018.

GIL, Antônio Carlos. Como elaborar projetos de pesquisa. São Paulo: Atlas, 2010. Acessado em: 24/05/2018.

JÚNIOR, F. Calvície feminina: classificação proposta. Disponível em: $<$ http://www.rbcp.org.br/details/62/calvicie-feminina--classificacao-proposta.$\quad$ Acessado em: $24 / 05 / 2018$

KLEINHANS, A. Stress e raiva em mulheres com alopecia androgenética. Disponível em: <http://www.bibliotecadigital.puc-campinas.edu.br/tde_arquivos/6/TDE-2012-03-23T052900Z1722/Publico/Andreia\%20Cristina\%20dos\%20Santos\%20Kleinhans.pdf>. Acessado em: 24/05/2018.

LAKATOS, E; MARCONI, M. Técnicas de pesquisa: Planejamento de pesquisas, amostragens e técnicas de pesquisa, elaboração, análise e interpretação de dados. $7^{\text {a }}$ ed. São Paulo: Atlas, 2011. Acessado em: 24/05/2018.

LIMA, J. et al. Tratamento de alopécia androgenética com o uso de laser de baixa potência e dermotonia (vacuoterapia) associado a argiloterapia e óleos essenciais. Disponível em: $<$ https://eventos.set.edu.br/index.php/sempesq/article/download/3976/1950>. Acessado em: 24/05/2018.

LINS, R. et al., Efeitos bioestimulantes do laser de baixa potência no processo de reparo. An Bras Dermatol. 2010;85(6):849-55. Disponível em: 〈http://www.scielo.br/pdf/abd/v85n6/v85n6a11.pdf>. Acessado em: 24/05/2018.

MEZZALIRA, J.; FREDERICO, I. Laserterapia de baixa intensidade - revisão de literatura. Bragança Paulista, 2007.2 Disponível em: <http://lyceumonline.usf.edu.br/salavirtual/documentos/1547.pdf>. Acessado em: 24/05/2018.

MULINARI-BRENNER, F.; SEIDEL, G.; HEPP, T. Entendendo a alopecia androgenética, Surgical \& Cosmetic Dermatology, vol. 3, núm. 4, 2011, pp. 329-337. Disponível em: $<$ http://www.redalyc.org/articulo.oa?id=265522077008>. Acessado em: 24/05/2018. 
REBELO, A.Novas estratégias para o tratamento da alopécia. Lisboa, 2015. Disponível em: $<$ http://recil.ulusofona.pt/bitstream/handle/10437/6180/TESE\%20FINAL.pdf?sequence=1>. Acessado em: 24/05/2018.

SANTOS, A.; ALMEIDA, T.; MOSER, D. Alopecia feminina: uma abordagem do processo e tratamentos não convencionais aplicados a esta patologia. Disponível em: <http://siaibib01.univali.br/pdf/Ana\%20Paula\%20Santos\%20e\%20Tatiana\%20Almeida.pdf>.

Acessado em: 24/05/2018.

SILVA, E.; PATRICIO, M.; PAULA, V. Terapia capilar para o tratamento da alopecia androgenética masculina e alopecia areata. Disponível em: <http://siaibib01.univali.br/pdf/Elaine\%20da\%20Silva,\%20Maiane\%20Patricio.pdf〉.

Acessado em: 24/05/2018.

SILVA, M.; SILVA, D. Abordagens da farmácia estética no tratamento da alopecia androgenética. Acessado em: 24/05/2018.

SILVA, R., Medicamentos antiandrógenos de uso oral para o tratamento da alopecia androgênica. Porto Alegre, 2011. Disponível em: <http://www.lume.ufrgs.br/bitstream/handle/10183/70120/000821952.pdf?sequence=1>. Acessado em: 24/05/2018.

WEIDE, A.; MILÃO, D. A utilização da Finasterida no Tratamento da Alopécia Androgenética. Disponível em: <http://revistaseletronicas.pucrs.br/ojs/index.php/graduacao/article/view/5035/3718>. Acessado em: 24/05/2018.

WIELEWSKI, C.; SERRÃO, C.; MOSER, D. Análise comparativa de técnicas de massagem utilizadas em um protocolo de tratamento para queda capilar. Disponível em: <http://siaibib01.univali.br/pdf/Camila\%20Wielewski,\%20Camilly\%20Serr\%C3\%A3o.pdf>.

Acessado em: 24/05/2018.

\section{Como citar este artigo (Formato ABNT):}

SILVA, Luciana Bárbara P. da; SANTOS, Belúzia Almeida. Uso do Laser de baixa intensidade no tratamento da Alopecia Androgenética: Uma Revisão Bibliográfica. Id on Line Revista Multidisciplinar e de Psicologia, 2018, vol.12, n.40, p. 1065-1081. ISSN: 1981-1179.

Recebido: 28.05.2018

Aceito: 29.05 .2018 